\title{
Combination of Strong MMP-2 and Weak TIMP-2 Immunostainings Is a Significant Prognostic Factor in Endometrial Carcinoma
}

\author{
Maria Honkavuori-Toivola, ${ }^{1,2}$ Markku Santala, ${ }^{1}$ Ylermi Soini, ${ }^{3,4}$ \\ Taina Turpeenniemi-Hujanen, ${ }^{2}$ and Anne Talvensaari-Mattila ${ }^{1}$ \\ ${ }^{1}$ Department of Obstetrics and Gynecology, Oulu University Hospital, University of Oulu, P.O. Box 5000, 90014 Oulu, Finland \\ ${ }^{2}$ Department of Oncology and Radiotherapy, Oulu University Hospital, University of Oulu, P.O. Box 5000, 90014 Oulu, Finland \\ ${ }^{3}$ Department of Pathology, Oulu University Hospital, University of Oulu, P.O. Box 5000, 90014 Oulu, Finland \\ ${ }^{4}$ Department of Pathology and Forensic Medicine, Institute of Clinical Medicine, Pathology and Forensic Medicine, School of Medicine, \\ University of Eastern Finland, Cancer Center of Eastern Finland, P.O. Box 1627, 70211 Kuopio, Finland
}

Correspondence should be addressed to Maria Honkavuori-Toivola; mhonkavu@paju.oulu.fi

Received 19 June 2013; Accepted 31 July 2013

Academic Editor: Sheng Pan

Copyright ( 2013 Maria Honkavuori-Toivola et al. This is an open access article distributed under the Creative Commons Attribution License, which permits unrestricted use, distribution, and reproduction in any medium, provided the original work is properly cited.

\begin{abstract}
Objective. The aim of this study was to evaluate the combined effects of MMP-2 and TIMP-2 protein immunoreactivities on the prognosis in endometrial carcinoma. Methods. Paraffin-embedded tissue samples from 225 primary endometrioid adenocarcinomas and 13 histologies other than endometrioid adenocarcinoma were immunohistochemically stained for MMP2 and TIMP-2. Results. In Kaplan-Meier analysis, the 5-year cancer-specific survival rate of the endometrioid adenocarcinoma patients with negative MMP-2 and positive TIMP-2 staining was $100 \%$, whereas only $78 \%$ of patients presenting with positive MMP2 and negative TIMP-2 staining results were alive at that time. In Cox regression analysis, patients with positive MMP-2 and negative TIMP-2 immunostaining had a 4.7-fold relative risk of death from endometrial carcinoma compared to the group of patients with negative MMP-2 and positive or negative TIMP-2 immunoreaction. Conclusions. MMP-2 seems to be the main metalloproteinase determining the prognosis in endometrial carcinoma. Combination of strong MMP-2 and weak TIMP-2 immunostainings was the most potent prognostic marker for poor survival.
\end{abstract}

\section{Introduction}

The extracellular matrix (ECM) is a dynamic fibrillar protein network of interacting macromolecules such as collagens, laminins, fibronectins, and proteoglycans. ECM components are degraded by different proteinases, the most prominent among which are the members of the matrix metalloproteinase (MMP) family $[1,2]$.

Matrix metalloproteinases are zinc-dependent endopeptidases that cleave the protein components of the extracellular matrix and thereby play a central role in tissue remodeling. There are 24 human MMPs known, and they are divided into two groups based on their cellular localization (secreted and membrane bound) or into five main groups according to their structure and substrate specificity: collagenases, gelatinases, stromelysins, matrilysins, and membrane-type MMPs (MT-MMPs) [3, 4]. MMPs can regulate the tumor microenvironment, and their expression and activation are increased in almost all human cancer types compared with normal tissue. In particular, MMP-2 and -9 have been linked to several malignant tumors [5-9].

MMP activity is tightly controlled by several endogenous inhibitors; the most thoroughly studied MMP inhibitors are the tissue inhibitors of metalloproteinases (TIMPs). The TIMP family consists of four members, TIMP-1, $-2,-3$, and -4 , that form noncovalent 1:1 stoichiometric complexes with MMPs. Although they all inhibit MMPs proteolytic activity, TIMPs differ in solubility, interaction with the proenzymes 
(proMMPs), affinity for the various MMPs, and regulation of expression [2, 10]. TIMP-2 is a unique member of TIMP family in the sense that, in addition to inhibiting MMPs, it selectively interacts with MT1-MMP to facilitate the cellsurface activation of pro-MMP-2. Thus, TIMP-2 functions both as an inhibitor of MMPs and is required for pro-MMP-2 activation [11].

A balance between MMP and TIMP activities is a prerequisite for normal function of an array of physiological processes. Disruption of the MMP-TIMP balance can result in a number of pathogenic processes including tumor invasion, metastasis, angiogenesis, and wound healing [1]. Increasing evidence suggests a much more complex role for TIMPs during tumor progression in addition to their regulation of MMP-mediated ECM degradation.

We have previously shown that high MMP-2 expression is associated with a poor survival in endometrial carcinoma [6]. In contrast, high TIMP-2 correlated with a favorable cancer-specific and overall survival in endometrial carcinoma patients [12]. It is still unknown which of the two, MMP-2 or TIMP-2, has superior effect on the prognosis of endometrial cancer patients. The aim of this study was to evaluate the effect of MMP-2 and TIMP-2 immunoreactive protein combinations on the prognosis of endometrial carcinoma. We also wanted to find out whether MMP-2 or TIMP-2 is a more powerful prognostic factor in endometrial cancer.

\section{Materials and Methods}

The study material consisted of 238 patients with histologically confirmed endometrial carcinoma whose paraffinembedded tumor tissue sections were available. Endometrial cancers were diagnosed between the years 1992 and 2000, and the patients were treated in the Department of Obstetrics and Gynecology, Oulu University Hospital. There were 225 endometrioid adenocarcinomas. The median age of these patients was 65 years at the time of the diagnosis (range 39-98). Their median body mass index (BMI) was $29 \mathrm{~kg} / \mathrm{m}^{2}$ (range 19-49). According to the International Federation of Gynecology and Obstetrics (FIGO) criteria, there were 149 stage I, 34 stage II, 37 stage III, and 5 stage IV cancers. Of the material, 117 tumors were well (grade 1), 75 moderately (grade 2 ), and 33 poorly (grade 3 ) differentiated. The median follow-up time was 77 months (range $0-136$ ).

In most cases, the primary treatments were extrafascial hysterectomy, bilateral salpingo-oophorectomy, and pelvic lymphadenectomy. Four patients had preoperative chemotherapy and 46 patients postoperative adjuvant cisplatinbased chemotherapy. Thirty patients received postoperative vaginal cuff brachytherapy and 90 patients postoperative external whole pelvic irradiation. Formalin-fixed, paraffinembedded endometrial tissue samples from the primary tumors were obtained from the files of the Department of Pathology of Oulu University Hospital.

In addition, there were 13 patients with histologies other than endometrioid adenocarcinoma, which were analyzed separately. Out of these 13 patients, three had adenoacanthoma, two had adenosquamous carcinoma, six had serous papillary carcinoma, and two had clear cell carcinoma.
2.1. Immunohistochemical Staining. Paraffin-embedded sections $(4 \mu \mathrm{m})$ from the primary tumors of endometrial carcinoma were stained using the avidin-biotin-immunoperoxidase technique as described previously $[6,12]$. A mouse monoclonal antibody to MMP-2 (CA-4001, Diabor Ltd., Oulu, Finland) or TIMP-2 (MAB 971, R\&D Systems, Minneapolis, MN, USA) was used as the primary antibody.

2.2. Evaluation of $M M P-2$ and TIMP-2 Immunostaining. For the study, the samples were evaluated by two independent observers blinded to the clinical data. The samples were considered as negative or positive according to the absence or presence of the immunoreaction for MMP-2 or TIMP2 protein in the cytoplasm of the tumor cells. The case was considered positive when $>1 \%$ of the neoplastic cells showed a positive staining for MMP-2 [6]. In TIMP-2 staining, the case was considered positive when $>25 \%$ of the tumor cells showed a positive immunoreaction [12].

2.3. Statistical Analysis. All statistical analyses were performed using the Statistical Package of Social Sciences software (SPSS, v. 17.0). The relationships between the clinicopathological categorical variables and MMP-2 or TIMP2 immunostaining were assessed with Fisher's exact test. Mann-Whitney $U$ test was used for continuous variables. Kaplan-Meier analysis was used to calculate the cancerspecific survival rates. The differences in survival between the subgroups were compared by a log-rank test. Cox backward regression model was used in multivariate analysis to assess the independency of the prognostic variables. Survival was defined as the time from the date of the diagnosis to the date of the last follow-up visit or death from endometrial cancer. $P$ values less than 0.05 were considered statistically significant.

\section{Results}

The positive immunoreaction for MMP-2 and TIMP-2 was seen in carcinoma cells as diffuse positive staining that seemed to localize in the cytoplasm in light microscopy (Figure 1). Different subgroups between endometrioid adenocarcinomas were formed in order to evaluate the prognostic role of MMP-2 and TIMP-2. The combinations and frequencies of different subgroups of MMP- 2 and TIMP- 2 are presented in Table 1.

Out of the 225 patients, 35 (16\%) died of endometrial carcinoma during the followup. The majority of the deaths ( $n=24,69 \%$ ) occurred in the subgroup where MMP2 was positive and TIMP-2 negative (22\%). Ten out of 93 (11\%) patients died of the disease in the subgroup where both MMP-2 and TIMP-2 were positive. In the subgroup with negative MMP-2 and positive TIMP-2 stainings, no deaths due to the disease were seen, whereas, in the subgroup where both MMP-2 and TIMP- 2 were negative, only one death was observed (6\%). A trend for a more favorable survival was observed in Kaplan-Meier analysis among the patients with negative MMP-2 immunostaining $(P=0.085)$ (Figure 2 ). The 5-year cancer-specific survival rate of the patients with negative MMP-2 and positive TIMP-2 stainings was $100 \%$. 


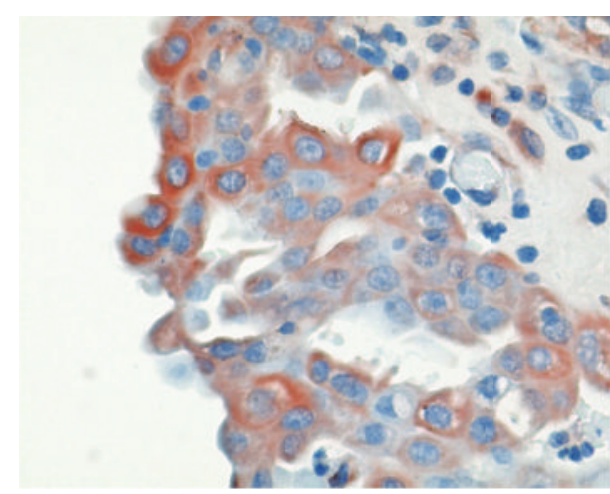

(a)

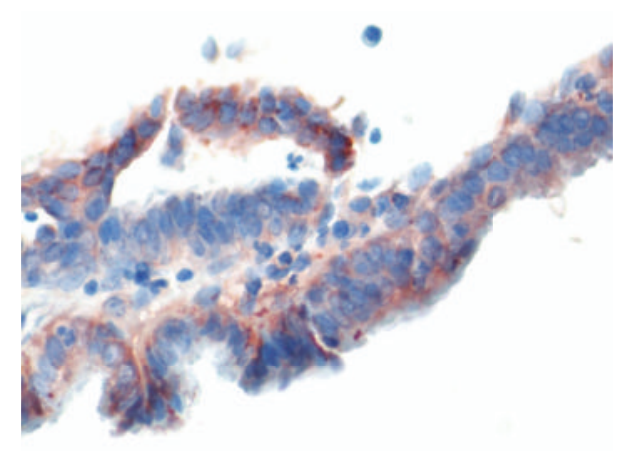

(c)

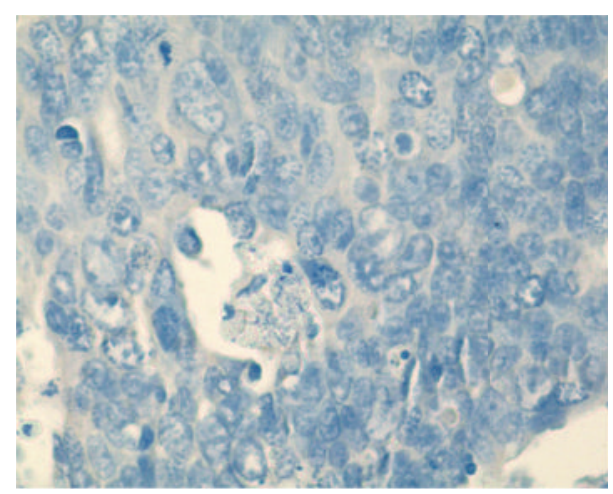

(b)

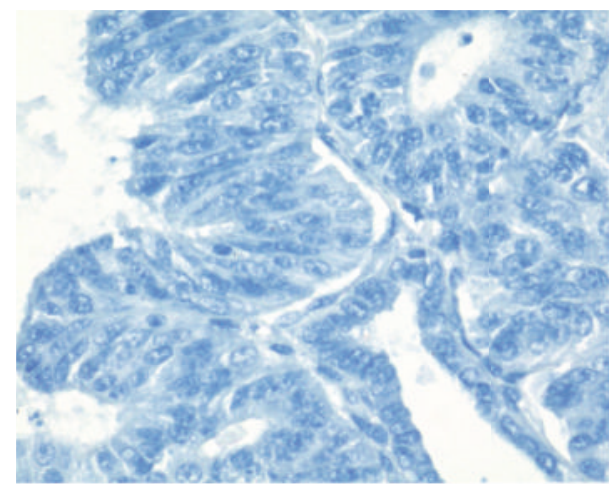

(d)

FIGURE 1: Positive and negative cytoplasmic immunostaining for MMP-2 and TIMP-2 in endometrioid adenocarcinoma. (a) represents positive staining of MMP-2, (b) negative staining of MMP-2, (c) positive staining of TIMP-2, and (d) negative staining of TIMP-2 (magnification $\times 320)$.

TABLE 1: The combinations and frequencies of MMP-2 and TIMP2 subgroups among endometrioid adenocarcinoma patients $(n=$ 225).

\begin{tabular}{lcc}
\hline Immunostaining & Frequency & Percent \\
\hline MMP-2- and TIMP-2+ & 6 & 2.7 \\
MMP-2- and TIMP-2- & 18 & 8.0 \\
MMP-2+ and TIMP-2+ & 93 & 41.3 \\
MMP-2+ and TIMP-2- & 108 & 48.0 \\
\hline Total & 225 & 100 \\
\hline
\end{tabular}

In contrast, only $78 \%$ of patients with positive MMP-2 and negative TIMP-2 immunoreactions were alive at that time. The corresponding figures for subgroups with MMP2-/TIMP-2- and MMP-2+/TIMP-2+ immunostainings were $93 \%$ and $90 \%$, respectively.

Since the number of patients in the two subgroups with negative MMP-2 immunostaining was small, the MMP-2 negative subgroups with either negative or positive TIMP2 staining were combined for further survival analyses. In Kaplan-Meier analysis the 5-year cancer-specific survival rate for the patients with negative MMP-2 immunostaining was 95\%. The difference in survival between the groups was statistically significant $(P=0.039)$ (Figure 3). Furthermore, when

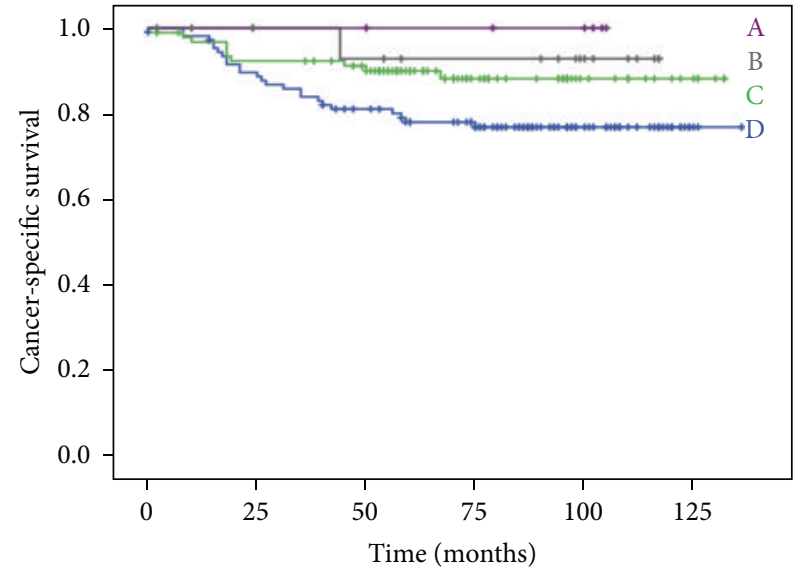
A: MMP-2- and TIMP-2+
C: MMP-2+ and TIMP-2+
B: MMP-2- and TIMP-2-
D: MMP-2+ and TIMP-2-

FIGURE 2: Effect of MMP-2 and TIMP-2 immunoreactive protein combinations on the cancer-specific survival in endometrioid adenocarcinoma patients.

only the MMP-2 positive patients were included in survival analyses, a difference in survival between these subgroups 


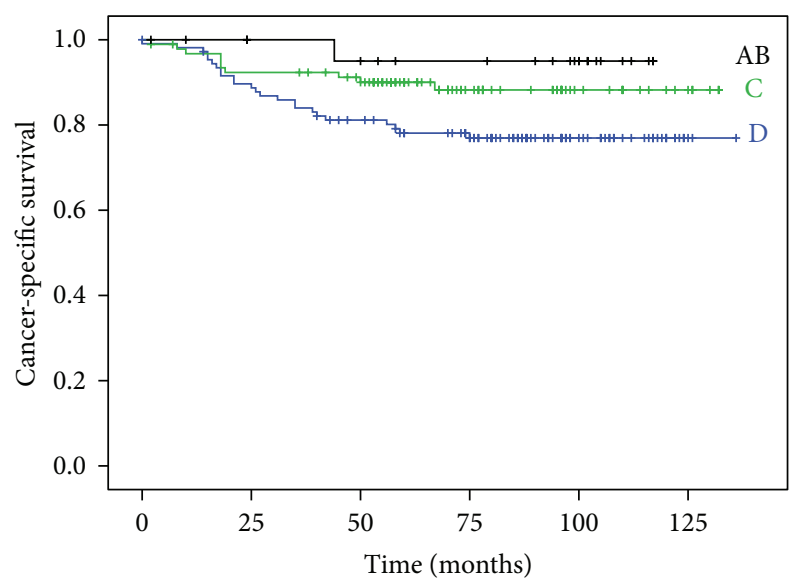

AB: MMP-2- and TIMP-2+ or TIMP-2-

C: MMP-2+ and TIMP-2+

D: MMP-2+ and TIMP-2-

FIgURE 3: Effect of MMP-2 and TIMP-2 immunoreactive protein combinations with MMP-2 negative subgroups combined in the cancer-specific survival in endometrioid adenocarcinoma patients.

with either negative or positive TIMP-2 immunostaining was also found $(P=0.048)$ (data not shown).

The value of prognostic variables, age, stage, grade, and MMP-2 and TIMP-2 immunostainings was evaluated by using the Cox regression multivariate analysis (Table 2). The MMP-2 and TIMP-2 immunostaining results were divided into three groups as described previously. The most significant prognostic factors for survival were the stage of the disease $(P<0.001)$ and histologic grade $(P=0.003)$. MMP2 and TIMP-2 also had a prognostic value in this analysis (cutoff $P<0.10$ ), although it did not quite reach statistical significance $(P=0.073)$. Patients with positive MMP-2 and negative TIMP-2 stainings had a 4.7-fold relative risk of death from endometrial carcinoma compared to the group of patients with negative MMP- 2 and positive or negative TIMP2 immunoreactions.

There were thirteen patients with a histology other than endometrioid adenocarcinoma, and both MMP-2 and TIMP2 staining results are available. The majority of them $(n=$ $10,77 \%$ ) showed a positive immunostaining for MMP-2 and were negative for TIMP-2 (Table 3 ). However, the number of histologies other than endometrioid adenocarcinoma was too small for more detailed analyses.

\section{Discussion}

Endometrial carcinoma is generally considered to have a good prognosis. However, the nature of the disease is heterogeneous, and there is a significant group of patients with a high risk of cancer recurrence and death. In this study, we were able to indicate a patient group with rather poor survival by using biological markers MMP-2 and TIMP-2.
TABLE 2: The independent and significant prognostic factors for the cancer-specific survival of endometrial cancer patients as evaluated by the Cox regression method. Relative risk of death, its $95 \%$ confidence interval, and $P$ values are given for each covariate. $n=$ 225.

\begin{tabular}{lcc}
\hline Variable & $\begin{array}{c}\text { Relative risk of } \\
\text { death }\end{array}$ & $\begin{array}{c}P \text { value to } \\
\text { remove }\end{array}$ \\
\hline Stage & 1 & $<0.001$ \\
I & $2.24(0.89-5.65)$ & \\
II & $3.65(1.65-8.09)$ & \\
III & $13.30(3.58-49.30)$ & \\
IV & & 0.003 \\
Grade & 1 & \\
1 & $2.91(1.23-6.86)$ & \\
2 & $4.98(1.98-12.50)$ & \\
3 & & \\
MMP-2 \& TIMP-2 & 1 & \\
MMP2- \& TIMP2- or + & \\
MMP2+ \& TIMP2+ & $2.30(0.29-18.34)$ & \\
MMP2+ \& TIMP2- & $4.70(0.63-35.00)$ & \\
\hline
\end{tabular}

We observed a combination of high MMP-2 and low TIMP2 expressions to identify a subgroup of women at high risk of poor outcome in endometrial carcinoma. Patients with negative MMP-2 immunostaining had the best prognosis, regardless of TIMP-2 staining result. Our results confirm our previous findings concerning the prognostic role of separately assessed MMP-2 and TIMP-2 immunoreactive proteins in endometrial carcinoma but also increase our knowledge about their mutual value in determining the prognosis in this cancer type $[6,12]$.

In this study, negative MMP-2 immunoreaction correlated with favorable survival and was superior to TIMP2 in determining the prognosis of endometrial carcinoma. Previous zymographic or immunohistochemical studies suggest that MMP-2 is the main metalloproteinase involved in the malignant behavior of endometrial cancer $[13,14]$. Positive MMP-2 immunostaining has been associated with aggressiveness and poor survival in various other cancer types including breast carcinoma [8], ovarian carcinoma [15], bladder cancer [16], and gastric [17] and colorectal cancers [18].

MMPs are under control of specific tissue inhibitors of metalloproteinases (TIMPs) at the posttranslational level. However, TIMPs are not simply inhibitors of proteolytic activity; they also have multifunctional roles, including cell growth, angiogenesis, and apoptosis [2]. TIMP-2 studies have given conflicting results concerning the relationship between TIMP-2 expression and prognosis. In endometrial carcinoma, there are only two studies that have included survival analyses, and they have shown somewhat contradictory results. Strong TIMP-2 expression has been associated with a favorable prognosis in our previous study, whereas, in a report by Moser et al., TIMP-2 was not associated with 
TABLE 3: MMP-2 and TIMP-2 immunostainings according to the histologic type among nonendometrioid carcinomas ( $n=13)$. Values are expressed as $n$.

\begin{tabular}{|c|c|c|c|c|c|}
\hline \multirow{2}{*}{ Immunostaining } & \multicolumn{4}{|c|}{ Histologic type } & \multirow{2}{*}{ Total } \\
\hline & Adenoacanthoma & Adenosquamous carcinoma & Serous papillary carcinoma & Clear cell carcinoma & \\
\hline MMP-2- and TIMP-2+ & 0 & 0 & 0 & 0 & 0 \\
\hline MMP-2- and TIMP-2- & 2 & 0 & 1 & 0 & 3 \\
\hline MMP-2+ and TIMP-2+ & 0 & 0 & 0 & 0 & 0 \\
\hline MMP-2+ and TIMP-2- & 1 & 2 & 5 & 2 & 10 \\
\hline
\end{tabular}

overall survival $[12,19]$. We consider cancer-specific survival to be a better method to analyze survival since it includes only deaths of the disease. Therefore, it is more relevant to a study evaluating associations of biological markers with the aggressiveness of a cancer.

Combined strong MMP-2 and weak TIMP-2 were more powerful prognostic factors than MMP-2 or TIMP-2 alone. The molar ratio of MMPs to TIMPs has been found to be higher in carcinoma tissues compared to nonneoplastic control tissues, which suggests the possibility of an imbalance in favor of the proteinases [20]. Thus, the effects of MMPs and their inhibitors depend on their relative proportions in local tumor microenvironment, that is why analyzing individual markers may not necessarily reveal the whole picture.

In Cox multivariate analysis, patients with positive MMP2 and negative TIMP- 2 stainings had an almost 5 -fold relative risk of dying from endometrial carcinoma compared to the group of patients with negative MMP-2 and positive or negative TIMP-2 immunoreactions. The range was rather wide, but also stage and grade had a wide range in Cox multivariate analysis. The conventional clinicopathological markers seem to be superior to MMP-2 and TIMP-2 in assessing the prognosis of endometrial carcinoma. It is possible, however, that MMP-2 and TIMP-2 expressions may add some further biological knowledge when assessing the prognosis of endometrial carcinoma patients.

The majority of histologies other than endometrioid adenocarcinoma showed positive immunoreaction for MMP2 and negative for TIMP-2. Type II tumors, consisting mainly of serous and clear cell carcinomas, are known to carry a higher mortality than type I tumors (mostly endometrioid adenocarcinomas). In our material, majority of adenoacanthomas showed negative MMP-2 and TIMP-2 immunostainings, which is not surprising because the prognosis of adenoacanthoma resembles the prognosis of endometrioid adenocarcinoma. In contrast, the majority of other histologies carrying poor prognosis were positive for MMP-2 and negative for TIMP-2. Our results suggest that the expression patterns of MMP-2 and TIMP-2 may be different in these two types of endometrial cancer. We conclude that high MMP-2 and low TIMP-2 expression levels may be linked to the aggressiveness of endometrial cancers. The number of nonendometrioid type tumors was, however, too small for further analyses. Increased MMP-2 expression has previously been associated with serous histology [21]. Graesslin et al. [22] are in line with our results who, reported lower TIMP2 expression levels in serous and clear cell carcinomas than in endometrioid adenocarcinoma. In contrast, Monaghan et al. observed significantly higher expressions of MMP-2 and MMP-9 in endometrioid tumors compared to serous tumor types [23]. We do not know the reason for the difference between the findings.

To our knowledge, this is the largest MMP-2 and TIMP2 immunohistochemistry study of endometrial carcinoma with a long followup published so far. Previously, Graesslin et al. [22, 24] have suggested high MMP-2 and low TIMP2 immunoreactive protein expressions to define a subgroup of endometrial tumors with a high risk of local and distant spread. The patient material in their studies was quite small, and no survival analyses were done. However, their findings are in line with our present data linking these biochemical markers to aggressiveness of endometrial cancer. Another strength of the present study is that all the patients were operated upon and treated in the same gynecological unit in a university hospital. All the patients were surgically staged according to the FIGO criteria and followed up systemically and for a sufficiently long time to observe the possible recurrences of the disease. Histological specimens were evaluated by two independent observers blinded to clinical data. However, evaluating immunohistochemical staining is always subjective to some extent. Death was relatively infrequent in our patient material, although the size of the study group was quite large. This may affect the results or at least makes it hard to establish differences between the subgroups. Another weakness of our study is the fact that the possible impact of treatment on survival could not be evaluated.

Taken together, patients with MMP-2 negative immunostaining have a rather good prognosis. In contrast, patients with MMP-2 positive staining form two groups with differing prognoses. The prognosis of patients with positive TIMP2 immunostaining is significantly better compared to the prognosis of patients with negative TIMP-2 staining. TIMP2 and especially MMP-2 may be useful for differentiating patients at low risk from those at high risk, and thus determining the need for adjuvant treatment.

\section{Conclusions}

Strong MMP-2 and weak TIMP-2 expression profiles define a subgroup of endometrial cancers with a more aggressive clinical course, suggesting that they may have prognostic potential. Moreover, MMP-2 seems to be superior to TIMP-2 
in determining the prognosis in endometrial cancer, if used separately.

\section{Acknowledgments}

The author would like to thank Mrs. Anne Bisi for her skillful technical assistance during this work. This study was supported in part by grants from the Cancer Society of Northern Finland and the Oulu University Scholarship Foundation.

\section{References}

[1] R. Chirco, X. W. Liu, K. K. Jung, and H. R. C. Kim, "Novel functions of TIMPs in cell signaling," Cancer and Metastasis Reviews, vol. 25, no. 1, pp. 99-113, 2006.

[2] E. Lambert, E. Dassé, B. Haye, and E. Petitfrère, "TIMPs as multifacial proteins," Critical Reviews in Oncology/Hematology, vol. 49, no. 3, pp. 187-198, 2004.

[3] D. Bourboulia and W. G. Stetler-Stevenson, "Matrix metalloproteinases (MMPs) and tissue inhibitors of metalloproteinases (TIMPs): positive and negative regulators in tumor cell adhesion," Seminars in Cancer Biology, vol. 20, no. 3, pp. 161-168, 2010.

[4] V. Şurlin, M. Ioana, and I. E. Pleşea, "Genetic patterns of metalloproteinases and their tissular inhibitors-clinicopathologic and prognostic significance in colorectal cancer," Romanian Journal of Morphology and Embryology, vol. 52, no. 1, pp. 231236, 2011.

[5] K. Sogawa, K. Kondo, H. Fujino et al., "Increased expression of matrix metalloproteinase- 2 and tissue inhibitor of metalloproteinase-2 is correlated with poor prognostic variables in patients with thymic epithelial tumors," Cancer, vol. 98, no. 9, pp. 1822-1829, 2003.

[6] M. Honkavuori, A. Talvensaari-Mattila, Y. Soini, T. Turpeenniemi-Hujanen, and M. Santala, "MMP-2 expression associates with CA 125 and clinical course in endometrial carcinoma," Gynecologic Oncology, vol. 104, no. 1, pp. 217-221, 2007.

[7] H. Ruokolainen, P. Pääkkö, and T. Turpeenniemi-Hujanen, "Expression of matrix metalloproteinase-9 in head and neck squamous cell carcinoma: a potential marker for prognosis," Clinical Cancer Research, vol. 10, no. 9, pp. 3110-3116, 2004.

[8] A. Talvensaari-Mattila, P. Pääkkö, and T. TurpeenniemiHujanen, "Matrix metalloproteinase-2 (MMP-2) is associated with survival in breast carcinoma," Teh British Journal of Cancer, vol. 89, no. 7, pp. 1270-1275, 2003.

[9] W. Sienel, J. Hellers, A. Morresi-Hauf et al., "Prognostic impact of matrix metalloproteinase-9 in operable non-small cell lung cancer," International Journal of Cancer, vol. 103, no. 5, pp. 647651, 2003.

[10] I. Stamenkovic, "Extracellular matrix remodelling: the role of matrix metalloproteinases," Journal of Pathology, vol. 200, no. 4, pp. 448-464, 2003.

[11] W. G. Stetler-Stevenson, “The tumor microenvironment: regulation by MMP-independent effects of tissue inhibitor of metalloproteinases-2," Cancer and Metastasis Reviews, vol. 27, no. 1, pp. 57-66, 2008.

[12] M. Honkavuori-Toivola, A. Talvensaari-Mattila, Y. Soini, T. Turpeenniemi-Hujanen, and M. Santala, "Immunoreactivity for
TIMP-2 is associated with a favorable prognosis in endometrial carcinoma," Tumour Biology, vol. 33, no. 4, pp. 935-941, 2012.

[13] K. Tamakoshi, F. Kikkawa, A. Nawa et al., "Characterization of extracellular matrix-degrading proteinase and its inhibitor in gynecologic cancer tissues with clinically different metastatic form," Cancer, vol. 76, no. 12, pp. 2565-2571, 1995.

[14] D. W. Park, H. S. Ryu, D. S. Choi, Y. H. Park, K. H. Chang, and C. K. Min, "Localization of matrix metalloproteinases on endometrial cancer cell invasion in vitro," Gynecologic Oncology, vol. 82, no. 3, pp. 442-449, 2001.

[15] A. A. Kamat, M. Fletcher, L. M. Gruman et al., "The clinical relevance of stromal matrix metalloproteinase expression in ovarian cancer," Clinical Cancer Research, vol. 12, no. 6, pp. 17071714, 2006.

[16] K. Vasala, P. Pääkkö, and T. Turpeenniemi-Hujanen, "Matrix metalloproteinase-2 immunoreactive protein as a prognostic marker in bladder cancer," Urology, vol. 62, no. 5, pp. 952-957, 2003.

[17] H. Alakus, G. Grass, J. K. Hennecken et al., "Clinicopathological significance of MMP-2 and its specific inhibitor TIMP-2 in gastric cancer," Histology and Histopathology, vol. 23, no. 8, pp. 917-923, 2008.

[18] M. Hilska, P. J. Roberts, Y. U. Collan et al., "Prognostic significance of matrix metalloproteinases-1, $-2,-7$ and -13 and tissue inhibitors of metalloproteinases-1, $-2,-3$ and -4 in colorectal cancer," International Journal of Cancer, vol. 121, no. 4, pp. 714723, 2007.

[19] P. L. Moser, L. Hefler, C. Tempfer, W. Neunteufel, D. G. Kieback, and G. Gitsch, "Immunohistochemical detection of matrix metalloproteinases (MMP) 1 and 2, and tissue inhibitor of metalloproteinase 2 (TIMP 2) in stage I and II endometrial cancer," Anticancer Research, vol. 19, no. 3, pp. 2365-2367, 1999.

[20] H. Ueno, K. Yamashita, I. Azumano, M. Inoue, and Y. Okada, "Enhanced production and activation of matrix metalloproteinase-7 (matrilysin) in human endometrial carcinomas," International Journal of Cancer, vol. 84, no. 5, pp. 470-477, 1999.

[21] R. Shaco-Levy, S. Sharabi, B. Piura, and N. Sion-Vardy, "MMP2 , TIMP-1, E-cadherin, and $\beta$-catenin expression in endometrial serous carcinoma compared with low-grade endometrial endometrioid carcinoma and proliferative endometrium," Acta Obstetricia et Gynecologica Scandinavica, vol. 87, no. 8, pp. 868874, 2008.

[22] O. Graesslin, A. Cortez, C. Uzan, P. Birembaut, C. Quereux, and E. Daraï, "Endometrial tumor invasiveness is related to metalloproteinase 2 and tissue inhibitor of metalloproteinase 2 expressions," International Journal of Gynecological Cancer, vol. 16, no. 5, pp. 1911-1917, 2006.

[23] H. Monaghan, N. MacWhinnie, and A. R. W. Williams, "The role of matrix metalloproteinases-2, -7 and -9 and $\beta$-catenin in high grade endometrial carcinoma," Histopathology, vol. 50, no. 3, pp. 348-357, 2007.

[24] O. Graesslin, A. Cortez, R. Fauvet, M. Lorenzato, P. Birembaut, and E. Daraï, "Metalloproteinase-2, -7 and -9 and tissue inhibitor of metalloproteinase-1 and -2 expression in normal, hyperplastic and neoplastic endometrium: a clinicalpathological correlation study," Annals of Oncology, vol. 17, no. 4, pp. 637-645, 2006. 


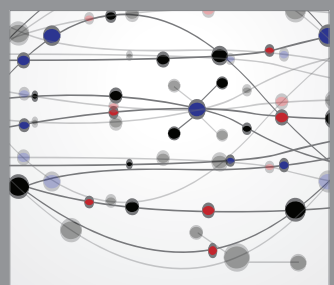

The Scientific World Journal
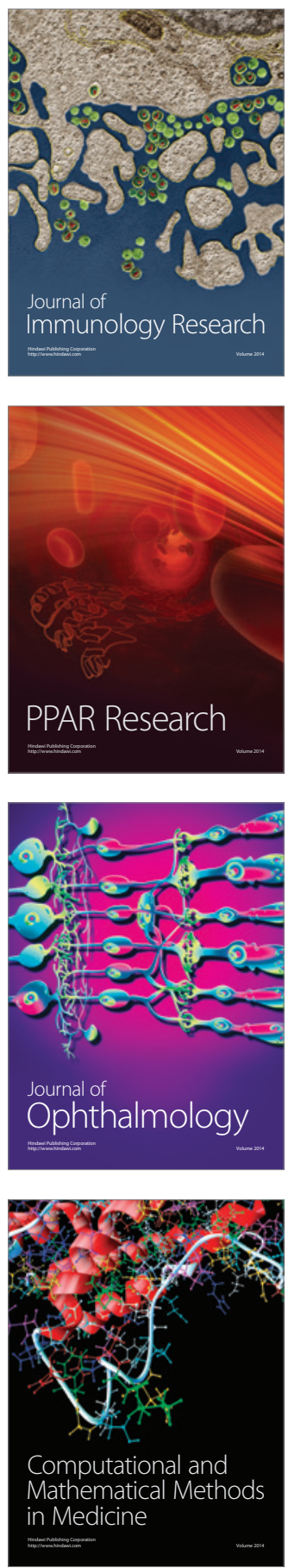

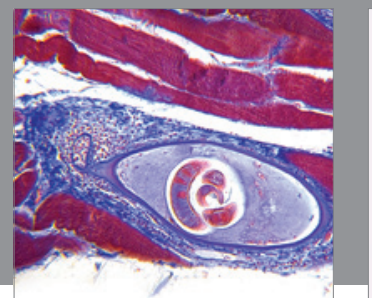

Gastroenterology

Research and Practice
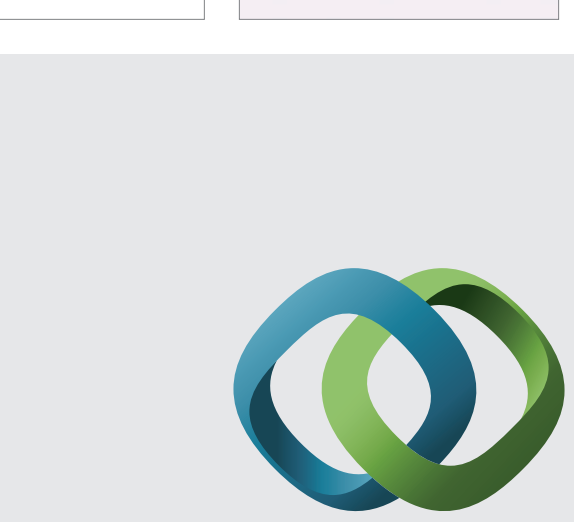

\section{Hindawi}

Submit your manuscripts at

http://www.hindawi.com
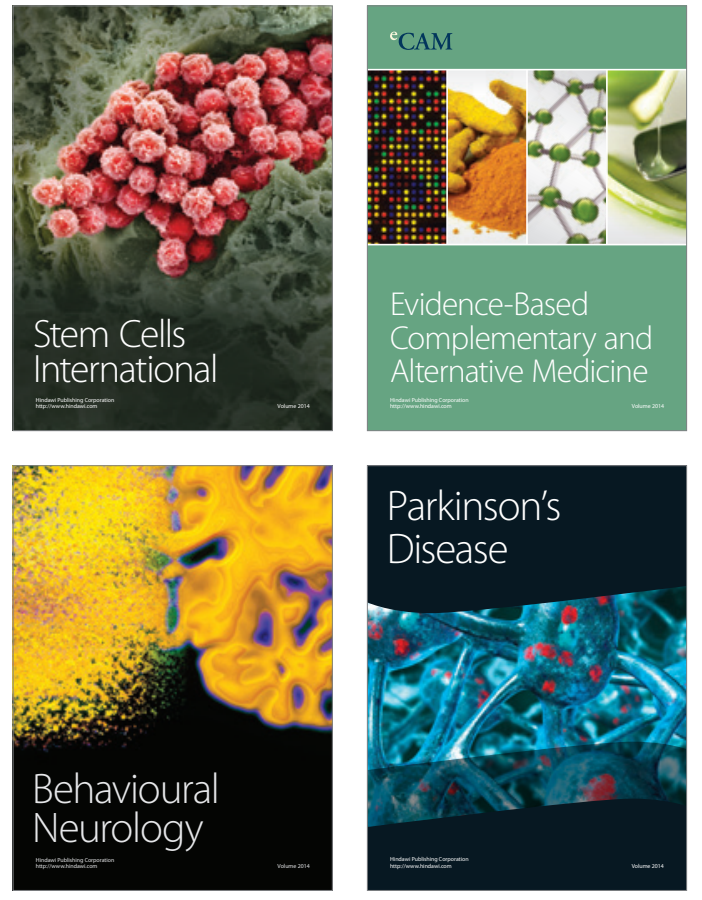
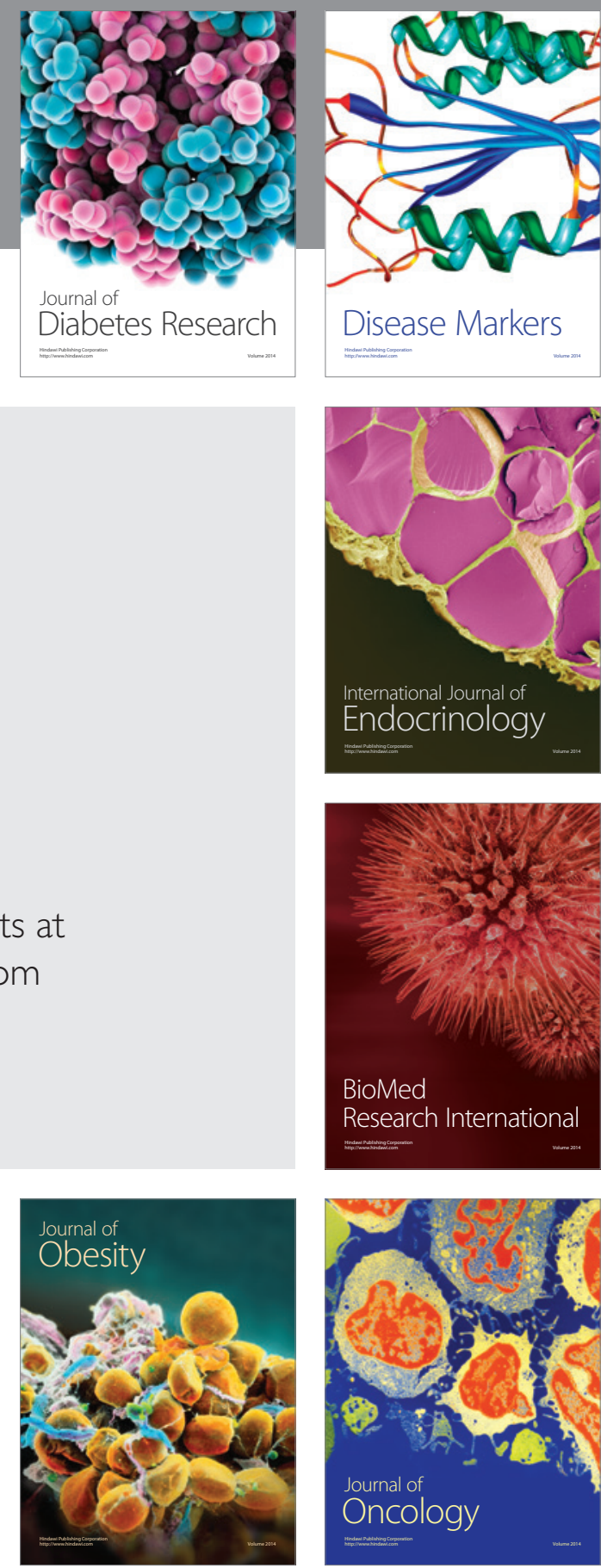

Disease Markers
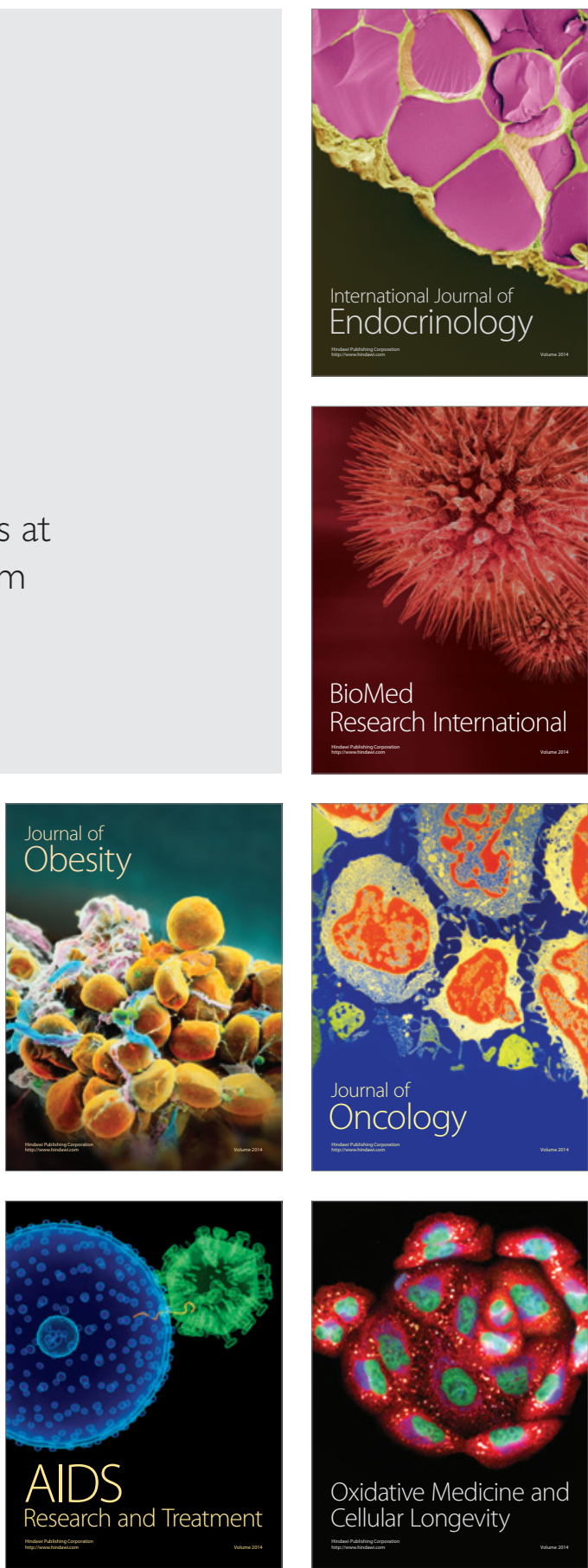